\title{
Evaluation of left atrial dysfunction by speckle tracking echocardiography in systolic and diastolic heart failure
}

\author{
Ahmed Ghanaym ${ }^{1,2}$, Khaled Elkhashab², Gomaa AbdelRazek2, Eman Mahmoud ${ }^{2}$ \\ ${ }^{1}$ Cardiology Department, Zagazig Insurance Institute, Zagazig; ${ }^{2}$ Cardiology Department, Faculty of Medicine, Fayoum \\ University, Faiyum, Egypt
}

\begin{abstract}
The study aimed to assess the accuracy of two-dimensional speckle tracking echocardiography (2DSTE) to evaluate the left atrial (LA) function in patients with heart failure. Additionally, if 2DSTE can differentiate accurately between heart failure preserved ejection fraction (HFpEF, HF with mid-range ejection fraction $(\mathrm{HFmrEF}=\mathrm{EF} 41-49 \%)$ and heart failure with reduced ejec-
\end{abstract}

Correspondence: Dr. Ahmad Ghanaym, Street 8, Building 28, Floor 7, Flat 28, Area 1a, Jabriya, Kuwait State.

Tel. +96.560010414. E-mail: D.ghanaym@yahoo.com

Key words: Speckle tracking echocardiography; systolic heart failure; diastolic heart failure; left atrial dysfunction.

Contributions: AG, KE, conception and design, administrative support; AG, GAR, provision of study materials or patients; AG, EM, data collection and assembly; AG, KE, GAR, EM, data analysis and interpretation, original draft preparation. All the authors read and approved the final version of the manuscript and agreed to be accountable for all aspects of the work.

Conflict of interest: The authors declare that they have no competing interests, and all authors confirm accuracy.

Funding: None declared.

Ethics approval and informed consent: The study protocol was approved by the local Research Ethics Committee, Fayoum

University, Egypt.

Received for publication: 30 September 2021.

Accepted for publication: 10 December 2021.

Publisher's note: All claims expressed in this article are solely those of the authors and do not necessarily represent those of their affiliated organizations, or those of the publisher, the editors and the reviewers. Any product that may be evaluated in this article or claim that may be made by its manufacturer is not guaranteed or endorsed by the publisher.

${ }^{\circ}$ Copyright: the Author(s), 2022

Licensee PAGEPress, Italy

Monaldi Archives for Chest Disease 2022; 92:2109

doi: 10.4081/monaldi.2022.2109

This article is distributed under the terms of the Creative Commons Attribution-NonCommercial International License (CC BY-NC 4.0) which permits any noncommercial use, distribution, and reproduction in any medium, provided the original author(s) and source are credited. tion fraction $(\mathrm{HFrEF}=\mathrm{EF} \leq 40 \%)$. The study included 186 patients of heart failure who were classified into 74 patients with HFpEF (LVEF $\geq 50 \%$ ), 56 patients with HFmrEF (LVEF 41-49\%), 56 patients with HFrEF (LVEF $<40 \%$ ), and 50 normal matched subjects. B-type natriuretic peptide (BNP) was more than $35 \mathrm{pg} / \mathrm{mL}$ for all patients. The conventional echocardiography evaluated left ventricle systolic and diastolic functions. The 2DSTE evaluated the LV global strain (LVGS), and strain and strain rate (SR) in each phase of LA function. LVGS was $-19.3 \pm 2.3 \%,-18.0 \pm 1.7 \%$, $16.1 \pm 2.0 \%$, and $-14.3 \pm 2.2$ in controls, HFpEF, and HFmrEF, and HFrEF, respectively $(\mathrm{p}<0.0001)$; GPALS was $34.1 \pm 6.7 \%$, $27.5 \pm 4.7 \%, 21.7 \pm 4.8 \%$ and $16.9 \pm 4.9 \%$ in controls, HFpEF, HFmrEF, HFrEF, respectively $(\mathrm{p}<0.0001)$; the GPACS was $14.8 \pm 4.3 \%, 12.3 \pm 2.2 \%, 9.7 \pm 2.3 \%$, and $7.5 \pm 2.6 \%$ in controls, HFpEF, HFmrEF, and HFrEF, respectively ( $p<0.0001)$; the PALSPACS was $19.4 \pm 3 \%, 15.1 \pm 4.4 \%, 12.0 \pm 3.4 \%$, and $9.3 \pm 3.3 \%$ in controls, HFpEF, HFmrEF, and HFrEF $(p<0.0001)$. Therefore, early LA dysfunction in heart failure can be detected accurately and easily by speckle tracking technique that could be a promising independent tool to better understand of heart failure and its classification.

\section{Introduction}

The left atrium plays an integral role in cardiac performance by modulating left ventricle (LV) filling [1]. Left atrial (LA) analysis by strain and strain rate imaging is based on both longitudinal and radial strain. However, current techniques do not have a sufficient resolution to measure the radial strain of the thin-walled LA; therefore, LA deformation assessment is only based on longitudinal strain, using the apical 4, 3 and 2 chamberviews [2]. The longitudinal strain curves for each segment of LA are generated automatically by software. These curves reflect the pathophysiology of atrial function [3]. Several studies have documented the prognostic role of peak-atrial longitudinal strain (PALS) in different clinical settings, including general population [4], myocardial infarction [5], aortic stenosis [6], and HFpEF [7]. Assessment of LA phasic function using 2D-speckle-tracking echocardiography (2D-STE) has gained considerable attention due to its high feasibility and reproducibility [8] and has led to the early detection of LA impairment in a number of conditions including HF [9]. Left ventricular ejection fraction (LVEF) is typically used to classify heart failure (HF) patients into HF with preserved LVEF $\geq 50 \%$ (HFpEF), HF with reduced LVEF $<40 \%$ (HFrEF) and HF with mid-range LVEF $40-49 \%$ (HFmrEF) according to European Society of Cardiology guidelines [10]. 
HFpEF patients constitute approximately more than half of the HF population [11]. Patients with HFmrEF account for approximately $10-20 \%$ of the HF population [12]. In patients with HFrEF, LA reservoir strain is strongly associated with estimated elevated filling pressure, impaired LV and right ventricle (RV) systolic function. Furthermore, it provides incremental prognostic information over LA volume, LV filling pressures, and GLS, so it allows powerful prognostication, independently of LA volume and left ventricular longitudinal contraction [13]. The aim of the study was to assess the accuracy of 2DSTE to evaluate the LA function in patients with heart failure. And can it differentiate accurately between HFpEF, HFmrEF and HFrEF?

\section{Materials and Methods}

\section{Study population}

Consecutive 186 patients from cardiology clinics in Fayoum University, Egypt, fulfilling HF recommendations of ESC at 2016 were prospectively enrolled in the study from February 2018 to January 2020. All patients were in optimal medical treatment and were hemodynamically stable. Inclusion criteria were patients in sinus rhythm, BNP more than $35 \mathrm{pg} / \mathrm{mL}$, and echocardiographic criteria of HFpEF $(n=74), \operatorname{HFmrEF}(n=56)$, and HFrEF $(n=56)[10,14]$. Exclusive criteria were any rhythm other than normal sinus rhythm, valvular heart diseases, congenital heart diseases, pacemaker insertion, pericardial diseases, patients with poor echocardiographic window and patients with renal or liver cell failure.

Fifty matched normal subjects with no history of medical diseases and normal echocardiography were selected from cardiology clinics in Fayoum University, Egypt to be included in the study. The study protocol was approved by the local Research Ethics Committee.

\section{Conventional echocardiography}

The standard echocardiographic Study was performed by one experienced sonographer, using a high-quality echocardiograph machine (Philips iE33). All subjects were in left-lateral decubitus position. The 2D, M-mode and Doppler techniques including tissue Doppler were performed to evaluate the left ventricular systolic and diastolic function [10]. LV volumes and LVEF were calculated using the modified biplane Simpson's method [14,15]. The trans-mitral E wave velocity in early diastole and the peak left ventricle filling velocity in late diastole (A wave) were estimated therefore E/A ratio was calculated automatically. An E/e' ratio was calculated as the ratio between $\mathrm{E}$ wave velocity and mean lateral and medial e' wave velocities (16). The LA volume at end systole would be maximum LAV (Max AV) therefore the Left atrium volume index (LAVI) was calculated by Max AV/BSA. (17). An LAVI cut off $>34 \mathrm{ml} / \mathrm{m}^{2}$ and E/e' ratio cut off $\geq 14$ were used as markers of LV diastolic dysfunction. The patients were classified accordingly [10].

\section{Speckle tracking echocardiography}

\section{Acquisition of image for longitudinal strain and strain rate}

Using conventional 2-D gray scale echocardiography and during breath hold with a stable ECG recording, the apical four, two and three-chamber views were obtained. The 2-D sector width was adjusted and care was taken to optimize visualization of the LV and LA cavity and to maximize LA area in apical views, avoiding foreshortening of the left atrium [18]. The frame rate was set between 60 and 80 frames per second and three consecutive cardiac cycles will be recorded and averaged.

\section{Left ventricular global strain}

Three points were anchored in the LV, apex and annular hinge points in apical 4, 3, 2 chamber views. The system will be allowed to process the data. After finishing tracing and auto processing the three views, the LV global strain will be obtained. Strain is the peak negative value that obtained at or before aortic valve closure [19].

\section{Left atrium strain and strain rate}

The machine software allowed off-line semiautomated analysis of speckle-based strain and strain rate. LA endocardial surface of each LA wall (septal, lateral, anterior and inferior walls) was manually traced in both 4 and 2 chamber views by a point andclick approach. An epicardial surface tracing was then automatically generated by the system, thus creating a region of interest (ROI) [20]. Automatically, the QRS onset is taken as a reference point, therefore enabling the measurement of PALS, corresponding to atrial reservoir. The $\mathrm{P}$ wave will be taken as the second reference point, therefore enabling the measurement of a first negative PALS corresponding to atrial systole, a second positive peak atrial strain, corresponding to LA conduit function, and their sum [21]. Lastly the software generated strain, strain rate curves to reflect the pathophysiology of LA phasic function [22]. The atrial function has three phases: The first is LA reservoir phase which is represented by GPALS and peak of left atrium strain rate at systole (LASRs), The second is LA pump function which is represented by GPACS and peak of left atrium strain rate at late diastole (LASRa), and the third is LA conduit phase which is represented by GPALS-PACS and peak of left atrium strain rate at early diastole (LASRe) [23].

\section{Statistical analysis}

The data were collected, organized, tabulated and statistically analyzed using SPSS software statistical computer package v. 18 (SPSS Inc, USA). The mean and standard deviation (SD) were calculated to qualitative the data. One-way ANOVA test was used as a test of significant. For qualitative data were presented as number and percentages, chi square $\left(\chi^{2}\right)$ was used to test significance of data. Pearson correlation was run to identify relation between different study parameters. Principle component analysis (PCA) was performed including all parameters of conventional and speckle tracking echocardiography. Varimax with Kaiser Normalization was chosen as a method for rotation. Coefficients $<0.4$ were excluded from analysis. Kaiser-Meyer-Olkin measure of sampling adequacy equals 0.838 while Bartlett's test of septicity was a statistically significant, $\mathrm{p}<0.0001$. Six components with eigenvalue more than one were extracted. After that, multivariate logistic regression analysis was conducted to determine the significant components as predictors for different types of HF. Components 6 was excluded from regression analysis because it contains 2 variables only. The receive operating characteristic (ROC) curve was used to determine the discrimination value of the different parameters for prediction of different conditions and to define the optimal cut-points for sensitivity and specificity. Significance of results was adopted at $\mathrm{p}<0.05$. 


\section{Results}

\section{Demographic, clinical and laboratory data}

Baseline characteristics of the study population were matched regarding age, gender, height, weight and body surface area (BSA). Also, no significant differences were found regarding smoking, HTN, DM, CAD, and hypercholesterolemia. The NYHA class ranged from I to IV was high significant in between the groups $(\mathrm{p}<0.0001)$. The BNP was $82 \pm 52.5$ in HFpEF, $202 \pm 154.4$ in HFmrEF, and $321 \pm 227$ in HFrEF with high significant $(\mathrm{p}<0.0001)$ (Table 1).

\section{Conventional echocardiography assessment}

LV dimensions (LVEDD and LVESD), LV volumes (LVEDS and LVESV), E/E' ratio and LAVI were significantly higher in HFrEF than these of HFmrEF which were significantly higher than these of HFpEF (Table 1).

\section{Speckle tracking echocardiography assessment}

LVGS, GPALS, LASRs, GPALS-PACS, LASRe, GPACS, and LASRa were significantly lower in HFrEF when compared to the corresponding values in HFmrEF which were significantly lower than the corresponding values in HFpEF which were significantly lower than the corresponding values in normal subjects (Table 1).

\section{Principle component analysis}

All parameters of conventional and speckle tracking echocardiography in HFpEF, HFmrEF, and HFrEF were extracted into six components. LVEF\%, LVGS and all 2DSTE parameters for left atrial phasic function were collected in the same component. The diastolic dysfunction parameters by conventional echocardiography were collected in three different components. (Table 2).

\section{Multivariate logistic regression analysis}

Component of LVEF\%, LVGS and all STE parameters for LA dysfunction was significantly predictor for all different types of HF. Whereas the component of diastolic dysfunction parameters obtained by conventional echocardiography including E/E ratio, LA diameter, LA area, MAX LAV and LAVI" was significantly predictive of HFpEF and HFmrEF (Table 3).

\section{Receiver operating characteristic (ROC) curves and cutoff point for $\mathrm{LV}$ systolic function and left atrial function}

The high sensitivity and specificity of LVGS and all STE parameters for LA dysfunction including GPALS, LASRs, GPALS-PACS, LASRe, GPACS and LASRa make them a novel predictor parameters to discriminate and early diagnose of HFpEF, HFmrEF and HFrEF (Table 4, Figure 1).

\section{Discussion}

Brain natriuretic peptide (BNP) is a cardiac hormone produced in the heart and an established biochemical marker for heart failure [24]. Stretching of ventricular cardiomyocytes is the most important stimulus of BNP regulation [25], but LV diastolic wall stress also reflects an increased BNP Therefore, BNP can be used in the diagnosis of HFpEF [26]. In our study the BNP level was significantly higher in HFrEF than HFmrEF which was high- er than HFpEF and these results was concordant to the study of Iwanaga et al. [25] and Modin et al. [27]. In our study, LVEDS and LVESV were significantly higher in HFrEF (LVEF $<40 \%$ ) than these of HFmrEF (LVEF 41-49\%) which were significantly higher than these of HFpEF (LVEF $>50 \%$ ). As known, chronically stressed LV leads to increase the tension of left ventricular wall causing remodeling and hypertrophy of the LV which lastly dilates [28]. When LV dysfunction occurs, both LVEDV and LVESV increase which in turn increase LV end-diastolic pressure [29] and this was concordant in our study. Several mechanisms that could be related to the development of HFpEF have been proposed. Previous studies [30] reported LV diastolic dysfunction and LV systolic longitudinal dysfunction, as shown by reduced longitudinal myocardial velocities and deformation, suggesting that DHF could be an HF stage preceding SHF [31,32]. In our study, the most patients of HFpEF had history of HTN $(68.9 \%)$, Zakeri et al. explained that in early stage hypertensive HFpEF, LA cardiomyocyte hypertrophy, titin hyperphosphorylation, and microvascular dysfunction occur in association with increased systolic and diastolic LA chamber stiffness, impaired atrioventricular coupling and decreased LV stroke volume [33]. In SHF, the left atrium is exposed to high LV filling pressures, thus the LA pressure rise to maintain adequate LV filling, and the rise in wall tension contributes to its enlargement. However, gradual increase in LA dimension disturb frank -starling relationship, decrease in atrial compliance and increase LA stiffness with decrease in LA reservoir function [34]. Our results demonstrated if LVEF \% was $29.1 \pm 6.9 \%$, the LAVI, LVGS, and GPALS would be $38.4 \pm 5.3 \mathrm{ml} / \mathrm{m} 2,-14.3$, and 16.9 respectively (Table 1). Study on systolic HF by Carluccio et al. ( $\mathrm{n}=454$ including 136 patients with LVEF $33 \%$ ) demonstrated if the LVEF was 33\%, the LAVI, LVGS, and GPALS would be $42.9 \pm 13.6 \mathrm{ml} / \mathrm{m}^{2},-10.4$, and $20.5 \%$, respectively [13]. The differences between the two studies could be explained by worse LVEF\%, lower number of patients, and exclusion of valvular heart diseases in our study compared to Carluccio et al.'s study. It was observed that LVGS, GPALS, LASRs, GPALS-PACS, LASRe, GPACS, and LASRa had high sensitivity, specificity and AUC to predict and early diagnose HFpEF, HFmrEF and HFrEF (Table 4, Figure 1). Furthermore, the component which include these parameters of 2DSTE was significantly predictor for all different types of HF (Tables 2 and $3)$. It is likely that intrinsic disorders with LA myocardial contractility as LA ischemia or fibrosis may play a role and mediated by increased work load imposed on the LA myocardium due to increased LV diastolic stress which overtime, will lead to intrinsic left atrial dysfunction and gradual decrease in LA contribution in LV filling [35]. These can be explained by the fact of heart failures were associated with the progressive conversion of the LA function from a storage and contractile chamber to a more passive-conduit chamber. Intrinsic alterations of LA myocardial contractility may play an important role. However, it is not clear that these myopathic changes happen firstly or occur lately as a consequence of LA enlargement and myofibrils stretching [36]. Many studies suggest that LV diastolic dysfunction and elevated filling pressure cannot completely account for LA dysfunction and that LA fibrosis may play an important role [37,38]. Atrial strain has been used also in transplanted patients to assess LV filling pressure [39]. Morris et al. suggested that LA dysfunction in HFpEF is likely to be related to the same fibrotic process, which influences the LV subendocardial layer secondary to several comorbidities such as diabetes mellitus, hypertension, and coronary artery disease [37]. The study by Al Saikhan et al. [29] compared the patients with $\operatorname{HFpEF}(n=110)$ and $\operatorname{HFmrEF}(n=61)$. 
Table 1. Characteristics of the study population including demographic, clinical, laboratory, conventional echocardiography, and speckle tracking echocardiography data.

\begin{tabular}{|c|c|c|c|c|c|c|}
\hline & & $\begin{array}{l}\text { Control } \\
(\mathrm{n}=50)\end{array}$ & $\begin{array}{l}\text { HFpEF } \\
(n=74)\end{array}$ & $\begin{array}{c}\text { HFmrEF } \\
(\mathrm{n}=56)\end{array}$ & $\begin{array}{l}\text { HFrEF } \\
(\mathrm{n}=56)\end{array}$ & $\begin{array}{l}\text { Overall } \\
\text { p-value }\end{array}$ \\
\hline Age (years) & & $58.0 \pm 6.2$ & $57.7 \pm 9.4$ & $57.8 \pm 9.8$ & $57.4 \pm 8.8$ & 0.999 \\
\hline Gender & $\begin{array}{l}\text { Female } \\
\text { Male }\end{array}$ & $\begin{array}{l}21(42) \\
29(58)\end{array}$ & $\begin{array}{l}34(45.9) \\
40(54.1)\end{array}$ & $\begin{array}{l}25(44.6) \\
31(55.4)\end{array}$ & $\begin{array}{l}27(48.2) \\
29(51.8)\end{array}$ & 0.933 \\
\hline Height $(\mathrm{cm})$ & & $170.3 \pm 9.1$ & $171.8 \pm 7.3$ & $173.1 \pm 7.7$ & $169.5 \pm 7.7$ & 0.074 \\
\hline Weight (kg) & & $78.1 \pm 8$ & $78.3 \pm 6.9$ & $78.0 \pm 7.8$ & $78 \pm 8.8$ & 0.977 \\
\hline $\operatorname{BSA}\left(m^{2}\right)$ & & $1.9 \pm 0.1$ & $1.9 \pm 0.1$ & $2 \pm 0.1$ & $1.9 \pm 0.1$ & 0.737 \\
\hline Smoking n, \% & $\begin{array}{l}\text { No } \\
\text { Yes }\end{array}$ & $\begin{array}{c}30(60) \\
20(40.0)\end{array}$ & $\begin{array}{c}37(50) \\
37(50.0)\end{array}$ & $\begin{array}{l}24(42.9) \\
32(57.1)\end{array}$ & $\begin{array}{l}31(55.4) \\
25(44.6)\end{array}$ & 0.318 \\
\hline Hypercholesterolemia n, \% & $\begin{array}{l}\text { No } \\
\text { Yes }\end{array}$ & $\begin{array}{c}50(100) \\
0(0.0)\end{array}$ & $\begin{array}{l}35(47.3) \\
39(52.7)\end{array}$ & $\begin{array}{l}26(46.4) \\
30(53.6)\end{array}$ & $\begin{array}{l}27(48.2) \\
29(51.8)\end{array}$ & 0.982 \\
\hline Diabetes mellitus n, \% & $\begin{array}{l}\text { No } \\
\text { Yes }\end{array}$ & $\begin{array}{c}50(100) \\
0(0.0)\end{array}$ & $\begin{array}{l}29(39.2) \\
45(60.8)\end{array}$ & $\begin{array}{l}23(41.1) \\
33(58.9)\end{array}$ & $\begin{array}{l}22(39.3) \\
34(60.7)\end{array}$ & 0.973 \\
\hline $\mathrm{CAD} n, \%$ & $\begin{array}{l}\text { No } \\
\text { Yes }\end{array}$ & $\begin{array}{c}50(100) \\
0(0.0)\end{array}$ & $\begin{array}{l}29(39.2) \\
45(60.8)\end{array}$ & $\begin{array}{l}18(32.1) \\
38(67.9)\end{array}$ & $\begin{array}{c}13(23.2) \\
43(76.8)\end{array}$ & 0.155 \\
\hline Arterial hypertension $\mathrm{n}, \%$ & $\begin{array}{l}\text { No } \\
\text { Yes }\end{array}$ & $\begin{array}{c}50(100) \\
0(0.0)\end{array}$ & $\begin{array}{l}23(31.1) \\
51(68.9)\end{array}$ & $\begin{array}{l}12(21.4) \\
44(78.6)\end{array}$ & $\begin{array}{l}10(17.9) \\
46(82.1)\end{array}$ & 0.185 \\
\hline NYHA & $\begin{array}{l}\text { I } \\
\text { II } \\
\text { III } \\
\text { IV }\end{array}$ & $\begin{array}{l}0(0.0) \\
0(0.0) \\
0(0.0) \\
0(0.0)\end{array}$ & $\begin{array}{c}53(71.6) \\
19(25.7) \\
2(2.7) \\
0(0.0)\end{array}$ & $\begin{array}{c}14(25.0) \\
34(60.7) \\
8(14.3) \\
0(0.0)\end{array}$ & $\begin{array}{c}2(3.6) \\
12(21.4) \\
17(30.4 \\
25(44.6)\end{array}$ & $<0.0001$ \\
\hline $\mathrm{BNP}(\mathrm{ng} / \mathrm{L})$ & & $18.4 \pm 5.7$ & $82 \pm 52.5$ & $202.2 \pm 154.4$ & $321.7 \pm 227$ & $<0.0001$ \\
\hline IVSD, $\mathrm{cm}$ & & $0.8 \pm 0.2$ & $0.9 \pm 0.3$ & $1.1 \pm 0.3$ & $1.2 \pm 0.2$ & $<0.0001$ \\
\hline LVPWD, $\mathrm{cm}$ & & $0.9 \pm 0.2$ & $0.9 \pm 0.2$ & $0.9 \pm 0.2$ & $1.1 \pm 0.2$ & $<0.0001$ \\
\hline IVSS, cm & & $1.2 \pm 0.3$ & $1.2 \pm 0.3$ & $1.3 \pm 0.2$ & $1.3 \pm 0.2$ & 0.002 \\
\hline LVPWS cm & & $1.3 \pm 0.5$ & $1.3 \pm 0.3$ & $1.1 \pm 0.3$ & $1.3 \pm 0.2$ & 0.006 \\
\hline LVEDD, cm & & $4.6 \pm 0.8$ & $4.7 \pm 0.8$ & $5 \pm 0.7$ & $6.6 \pm 0.7$ & $<0.0001$ \\
\hline LVEDS, cm & & $2.8 \pm 0.4$ & $3.2 \pm 0.6$ & $4 \pm 0.6$ & $5.4 \pm 0.7$ & $<0.0001$ \\
\hline $\mathrm{FS}, \%$ & & $39.6 \pm 5.2$ & $31.7 \pm 7$ & $20.6 \pm 2.7$ & $18.1 \pm 3,9$ & $<0.0001$ \\
\hline LVEDV, ml ${ }^{b}$ & & $107.1 \pm 27.4$ & $108 \pm 27.3$ & $113.8 \pm 19.5$ & $156.3 \pm 20.6$ & $<0.0001$ \\
\hline LVESV, ml ${ }^{b}$ & & $39.6 \pm 13.3$ & $45.5 \pm 12.6$ & $62.8 \pm 11.7$ & $111.1 \pm 18.8$ & $<0.0001$ \\
\hline LVEF\%ob & & $63 \pm 6.4$ & $57.7 \pm 4.8$ & $45 \pm 2.8$ & $29.1 \pm 6.9$ & $<0.0001$ \\
\hline E wave, $\mathrm{cm} / \mathrm{s}$ & & $90.6 \pm 16.2$ & $100.1 \pm 18.5$ & $109.4 \pm 22.1$ & $122.2 \pm 25.1$ & $<0.0001$ \\
\hline $\mathrm{A}(\mathrm{cm} / \mathrm{s})$ & & $66.2 \pm 15.2$ & $79.9 \pm 21.9$ & $73.9 \pm 22$ & $76.4 \pm 25.1$ & $<0.0001$ \\
\hline E/A ratio & & $1.4 \pm 0.3$ & $1.3 \pm 0.3$ & $1.5 \pm 0.3$ & $1.7 \pm 0.5$ & $<0.0001$ \\
\hline Medial e' & & $9.9 \pm 2.1$ & $6.6 \pm 1.7$ & $6.8 \pm 1.6$ & $6.7 \pm 2.3$ & $<0.0001$ \\
\hline Lateral e' & & $12.6 \pm 2.9$ & $8.1 \pm 2.3$ & $8.1 \pm 2$ & $8.3 \pm 2.3$ & $<0.0001$ \\
\hline E/e' ratio & & $8.5 \pm 2.1$ & $14.6 \pm 3.6$ & $15.3 \pm 3.5$ & $17.6 \pm 4.8$ & $<0.0001$ \\
\hline LA diameter $\mathrm{cm}^{\mathrm{a}}$ & & $3.3 \pm 0.6$ & $3.8 \pm 0.8$ & $3.8 \pm 0.7$ & $3.7 \pm 0.7$ & $<0.0001$ \\
\hline LA area mm² & & $18.7 \pm 3.5$ & $21.1 \pm 3$ & $23.6 \pm 2.5$ & $23.7 \pm 4$ & $<0.0001$ \\
\hline MAX LAV ml & & $52.8 \pm 13.8$ & $69.1 \pm 9.2$ & $74.2 \pm 7.6$ & $73.8 \pm 10.7$ & $<0.0001$ \\
\hline LAVi mL/m² & & $24.6 \pm 4.6$ & $36.0 \pm 4.6$ & $37.4 \pm 4.4$ & $38.4 \pm 5.3$ & $<0.0001$ \\
\hline Diastolic dysfunction grading & $\begin{array}{l}\text { I } \\
\text { II } \\
\text { III }\end{array}$ & $\begin{array}{l}0 \\
0 \\
0\end{array}$ & $\begin{array}{c}35(47.3) \\
33(44.6) \\
6(8.1)\end{array}$ & $\begin{array}{c}24(42.9) \\
25(44.6) \\
7(12.5)\end{array}$ & $\begin{array}{l}19(33.9) \\
23(41.1) \\
14(25.0)\end{array}$ & 0.092 \\
\hline LVGS, \% & & $-19.3 \pm 2.3$ & $-18.0 \pm 1.7$ & $-16.1 \pm 2.0$ & $-14.3 \pm 2.2$ & $<0.0001$ \\
\hline GPALS, $\%$ & & $34.1 \pm 6.7$ & $27.5 \pm 4.7$ & $21.7 \pm 4.8$ & $16.9 \pm 4.9$ & $<0.0001$ \\
\hline LASRs & & $1.2 \pm 0.2$ & $1.1 \pm 0.2$ & $0.9 \pm 0.2$ & $0.7 \pm 0.1$ & $<0.0001$ \\
\hline GPALS - PACS, $\%$ & & $19.4 \pm 3.0$ & $15.1 \pm 4.4$ & $12.0 \pm 3.4$ & $9.3 \pm 3.3$ & $<0.0001$ \\
\hline LASRe & & $1.1 \pm 0.2$ & $0.9 \pm 0.2$ & $0.9 \pm 0.2$ & $0.8 \pm 0.2$ & $<0.0001$ \\
\hline GPACS, $\%$ & & $14.8 \pm 4.3$ & $12.3 \pm 2.2$ & $9.7 \pm 2.3$ & $7.5 \pm 2.6$ & $<0.0001$ \\
\hline LASRa & & $1.6 \pm 0.3$ & $1.1 \pm 0.3$ & $0.8 \pm 0.3$ & $0.7 \pm 0.3$ & $<0.0001$ \\
\hline
\end{tabular}

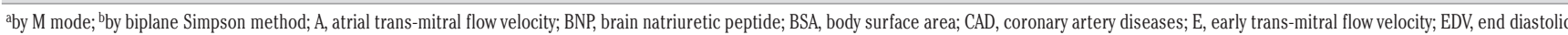
volume; ESV, end systolic volume; FS, fraction shortening; GPACS, global peak atrial contraction strain; GPALS, global peak atrial longitudinal strain; GPALS-PACS, refers to LA longitudinal strain at end of atrial contraction; HFmrEF, heart failure with mid-range ejection fraction; HFpEF, Heart failure with preserved ejection fraction; HFrEF, heart failure with reduced ejection fraction; IVSD, interventricular septum at diastole; IVSS, inter-ventricular septum at systole; LA, left atrium; LASRs, left atrial strain rate at systole; LASRe, peak of left atrial strain rate at early diastole; LASRa, peak of left atrial strain rate at late diastole; LAVi, LA volume indexed; LVEDD, left ventricular end diastolic diameter; LVEDS, left ventricular end systolic diameter; LVEF \%, Left ventricular ejection fraction; LVPWD, left ventricular posterior wall diameter diastole; LVPWS, left ventricular posterior wall diameter systole; Max AV, maximum atrial volume; just before the opening of mitral valve; NYHA, New York Heart Association. 
Table 2. Principle component analysis (PCA) for conventional and 2DSTE parameters in all types of heart failure.

Component of LV systolic function, and LA phasic function by speckle tracking echocardiography.

\begin{tabular}{lc} 
FS, \% & 0.696 \\
LVEF, \% & 0.779 \\
LVGS, \% & 0.7278 \\
GPALS, \% & 0.885 \\
LASRs & 0.633 \\
GPALS-PACS, \% & 0.811 \\
LASRe & 0.653 \\
GPACS, \% & 0.774 \\
LASRa & 0.644 \\
IVSD, cm & 0.780 \\
LVPWD, cm & 0.842 \\
IVSS, cm & 0.835 \\
LVPWS, cm & 0.788 \\
LVEDD, cm & 0.906 \\
LVEDS, cm & 0.797 \\
LVEDV, ml & 0.897 \\
LVESV, ml & 0.780 \\
E/E ratio & 0.646 \\
LA diameter, cm & 0.802 \\
LA area, mm & 2 \\
Max LAV, ml & 0.799 \\
LAVI, mL/m ${ }^{2}$ & 0.775 \\
E wave, cm/s & 0.723 \\
Medial E & 0.576 \\
Lateral E & 0.801 \\
A wave, cm & 0.804 \\
E/A ratio & 0.939 \\
\hline
\end{tabular}

Component of systolic and diastolic LV walls.

Component of systolic and diastolic LV dimensions and volumes.

Diastolic dysfunction component including parameters of LA enlargement and E/E ratio. E/A ratio

A, atrial trans-mitral flow velocity; E, early trans-mitral flow velocity; EDV, end diastolic volume; ESV, end systolic volume; FS, fraction shortening; GPACS, Global peak atrial contraction strain; GPALS, Global peak atrial longitudinal strain; GPALS-PACS, refers to LA longitudinal strain at end of atrial contraction; IVSD, interventricular septum at diastole; IVSS, inter-ventricular septum at systole; LA, left atrium; LASRs, left atrial strain rate at systole; LASRe, peak of left atrial strain rate at early diastole; LASRa, peak of left atrial strain rate at late diastole; LAVI, LA volume indexed; LVEDD, left ventricular end diastolic diameter; LVEDS, left ventricular end systolic diameter; LVEF \%, left ventricular ejection fraction; LVGS, LV global strain; LVPWD, left ventricular posterior wall diameter diastole; LVPWS, left ventricular posterior wall diameter systole; Max AV, maximum atrial volume; just before the opening of mitral valve.

Table 3. Multivariate logistic regression showing the significant components* for prediction of different types of heart failure.

\begin{tabular}{|c|c|c|c|c|}
\hline \multirow[t]{2}{*}{ Extracted components } & \multirow[t]{2}{*}{ p-value } & \multirow[t]{2}{*}{$\begin{array}{l}\text { Odds } \\
\text { ratio }\end{array}$} & \multicolumn{2}{|c|}{$\begin{array}{l}95 \% \text { CI } \\
\text { for OR }\end{array}$} \\
\hline & & & Lower & Upper \\
\hline \multicolumn{5}{|l|}{ HFPEF } \\
\hline 1. Component of LV systolic function, and LA phasic function by speckle tracking echocardiography. & $<0.001$ & 2.525 & 1.639 & 3.891 \\
\hline 2. Component of systolic and diastolic LV dimensions and volumes. & 0.046 & 0.708 & 0.505 & 0.993 \\
\hline 3. Component of systolic and diastolic LV walls. & $<0.001$ & 0.240 & 0.144 & 0.401 \\
\hline 4. Diastolic function component including parameters of LA enlargement and E/E ratio. & 0.003 & 1.797 & 1.227 & 2.631 \\
\hline 5. Diastolic function component including early diastolic filling velocities. & $<0.001$ & 0.352 & 0.234 & 0.530 \\
\hline Constant & $<0.001$ & 0.245 & & \\
\hline \multicolumn{5}{|l|}{ HFmrEF } \\
\hline 1. Component of LV systolic function, and LA phasic function by speckle tracking echocardiography. & $<0.001$ & 0.353 & 0.222 & 0.562 \\
\hline 2. Component of systolic and diastolic LV dimensions and volumes. & 0.250 & 0.826 & 0.596 & 1.144 \\
\hline 3. Component of systolic and diastolic LV walls. & $<0.001$ & 0.563 & 1.398 & 0.797 \\
\hline 4. Diastolic function component for LA enlargement and E/E ratio. & $<0.001$ & 1.014 & 1.382 & 2.937 \\
\hline 5. Diastolic function component for early diastolic filling velocities. & 0.708 & 0.935 & 1.660 & 1.326 \\
\hline Constant & $<0.001$ & 0.207 & & \\
\hline \multicolumn{5}{|l|}{ HFrEF } \\
\hline 1. Component of LV systolic function, and LA phasic function by speckle tracking echocardiography & $<0.001$ & 0.009 & 0.001 & 0.078 \\
\hline 2. Component of systolic and diastolic LV dimensions and volumes. & 0.013 & 5.291 & 1.426 & 19.627 \\
\hline 3. Component of systolic and diastolic LV walls. & $<0.001$ & 293.614 & 23.912 & 3605.294 \\
\hline 4. Diastolic function component for LA enlargement and E/E ratio. & 0.060 & 3.414 & 0.979 & 12.52 \\
\hline 5. Diastolic function component for early diastolic filling velocities. & 0.725 & 0.839 & 0.316 & 2.227 \\
\hline Constant & $<0.001$ & 0.004 & & \\
\hline
\end{tabular}

*Component number 6 in PCA was excluded from Multivariate regression analysis because it contains 2 variables only. 
The mean average of GPALS (26.2 in HFpEF and 20.6 in HFmrEF), GPACS (13.1 in HFpEF and 9.8 in HFmrEF), and GPALS-PACS (13.0 in HFpEF and 10.7 in HFmrEF) were similar to our results; GPALS (27.5 in HFpEF and $21.7 \%$ in HFmrEF), GPACS (12.3 in HFpEF and 9.7 in HFmrEF), and GPALS-PACS (15.1 in HFpEF and 12.0 in HFmrEF) And similar to our study, the worst of LVEF was associated with worse LA functions. These slightly differences can be explained firstly by higher patient's numbers of HFpEF and HFmrEF of that study than our study, secondly higher mean of LVEF which was $64.9 \%$ versus $57.7 \%$ in $\mathrm{HFpEF}$ and $44.9 \%$ versus $45 \%$ in HFmrEF in our study [29]. In our study, it was observed that the component of LVEF, LVGS and LA dysfunction which obtained by 2DSTE had high significance prediction for all type of HF. on the other hand the component of LA dysfunction which obtained by conventional echocardiography had significance prediction in HFpEF and HFmrEF only, and this could be explained by LV fibrosis and restricted mitral annular motion in HFrEF make E/e' unreliable for quantifying LV diastolic pressure [40].

Table 4. Receiver operating characteristic (ROC) curves and cutoff point for LV systolic function and left atrial function in HFpEF, HFmrEF, HFrEF, and controls.

\begin{tabular}{|c|c|c|c|c|c|c|c|}
\hline \multirow[t]{2}{*}{ STE parameters } & \multirow[t]{2}{*}{ LVGS \% } & \multicolumn{2}{|c|}{ LA reservoir phase } & \multicolumn{2}{|c|}{ LA conduit phase } & \multicolumn{2}{|c|}{ LA pump function } \\
\hline & & GPALS & LASRs & GPALS - PACS & LASRe & GPACS & LASRa \\
\hline \multicolumn{8}{|c|}{ HFpEF versus control groups } \\
\hline AUC & 0.663 & 0.792 & 0.673 & 0.769 & 0.775 & 0.662 & 0.883 \\
\hline$p$-value & 0.002 & $<0.0001$ & 0.001 & $<0.0001$ & $<0.0001$ & 0.002 & $<0.0001$ \\
\hline Cut-off point & -20.5 & 34.5 & 1.3 & 15.5 & 1.2 & 15.5 & 1.2 \\
\hline Sensitivity & 89.2 & 95.9 & 94.6 & 60.8 & 91.9 & 93.3 & 63.5 \\
\hline Specificity & 40.0 & 56.0 & 48.0 & 84.0 & 54.0 & 44.0 & 98.0 \\
\hline \multicolumn{8}{|c|}{ HFpEF versus HFmrEF } \\
\hline AUC & 0.768 & 0.792 & 0.738 & 0.712 & 0.487 & 0.798 & 0.686 \\
\hline$p$-value & $<0.0001$ & $<0.0001$ & $<0.0001$ & $<0.0001$ & 0.800 & $<0.0001$ & $<0.0001$ \\
\hline Cut-off point & -15.5 & 19.5 & 1.1 & 9.5 & 1.1 & 10.5 & 0.6 \\
\hline Sensitivity & 44.6 & 55.4 & 85.7 & 33.9 & 83.9 & 69.7 & 35.7 \\
\hline Specificity & 100.0 & 100.0 & 59.5 & 98.6 & 29.7 & 79.7 & 94.6 \\
\hline \multicolumn{8}{|c|}{ HFpEF versus HFrEF } \\
\hline AUC & 0.899 & 0.907 & 0.929 & 0.842 & 0.654 & 0.928 & 0.777 \\
\hline P-value & $<0.0001$ & $<0.0001$ & $<0.0001$ & $<0.0001$ & 0.003 & $<0.0001$ & $<0.0001$ \\
\hline Cut-off point & -15.5 & 19.5 & 1.0 & 9.5 & 0.8 & 9.5 & 0.6 \\
\hline Sensitivity & 62.5 & 71.4 & 100.0 & 60.7 & 57.1 & 82.1 & 48.2 \\
\hline Specificity & 100.0 & 100.0 & 75.7 & 98.6 & 68.9 & 91.9 & 100.0 \\
\hline \multicolumn{8}{|c|}{ HFmrEF versus control } \\
\hline AUC & 0.847 & 0.917 & 0.818 & 0.933 & 0.785 & 0.847 & 0.959 \\
\hline $\mathrm{p}$-value & $<0.0001$ & $<0.0001$ & $<0.0001$ & $<0.0001$ & $<0.0001$ & $<0.0001$ & $<0.0001$ \\
\hline Cut-off point & -17.5 & 30.0 & 1.1 & 16.5 & 1.1 & 12.5 & 1.2 \\
\hline Sensitivity & 78.6 & 100.0 & 89.3 & 87.5 & 83.9 & 85.7 & 82.1 \\
\hline Specificity & 72.0 & 66.0 & 66.0 & 80.0 & 68.0 & 66.0 & 98.0 \\
\hline \multicolumn{8}{|c|}{ HFmrEF versus HFrEF } \\
\hline AUC & 0.702 & 0.774 & 0.793 & 0.732 & 0.682 & 0.737 & 0.640 \\
\hline p-value & $<0.0001$ & $<0.0001$ & $<0.0001$ & $<0.0001$ & 0.001 & $<0.0001$ & 0.011 \\
\hline Cut-off point & -13.5 & 16.5 & 1.0 & 7.5 & 0.7 & 6.5 & 0.4 \\
\hline Sensitivity & 41.1 & 60.7 & 96.4 & 41.1 & 37.5 & 39.3 & 33.9 \\
\hline Specificity & 96.4 & 89.3 & 53.6 & 100.0 & 100.0 & 100.0 & 94.6 \\
\hline \multicolumn{8}{|c|}{ HFrEF versus controls } \\
\hline AUC & 0.940 & 0.974 & 0.949 & 0.984 & 0.871 & 0.931 & 0.972 \\
\hline $\mathrm{p}$-value & $<0.0001$ & $<0.0001$ & $<0.0001$ & $<0.0001$ & $<0.0001$ & $<0.0001$ & $<0.0001$ \\
\hline Cut-off point & -16.5 & 26.5 & 1.0 & 14.5 & 0.9 & 10.5 & 1.2 \\
\hline Sensitivity & 80.4 & 100.0 & 96.4 & 91.1 & 82.1 & 91.1 & 85.7 \\
\hline Specificity & 90.0 & 84.0 & 86.0 & 92.0 & 80.0 & 78.0 & 98.0 \\
\hline
\end{tabular}

AUC, area under curve; GPALS, global peak atrial longitudinal strain; GPALS-PACS, refers to LA longitudinal strain at end of atrial contraction; GPACS, global peak atrial contraction strain; LA, left atrium; LASRa, peak of left atrial strain rate at late diastole; LASRe, peak of left atrial strain rate at early diastole; LASRs, left atrial strain rate at systole. 


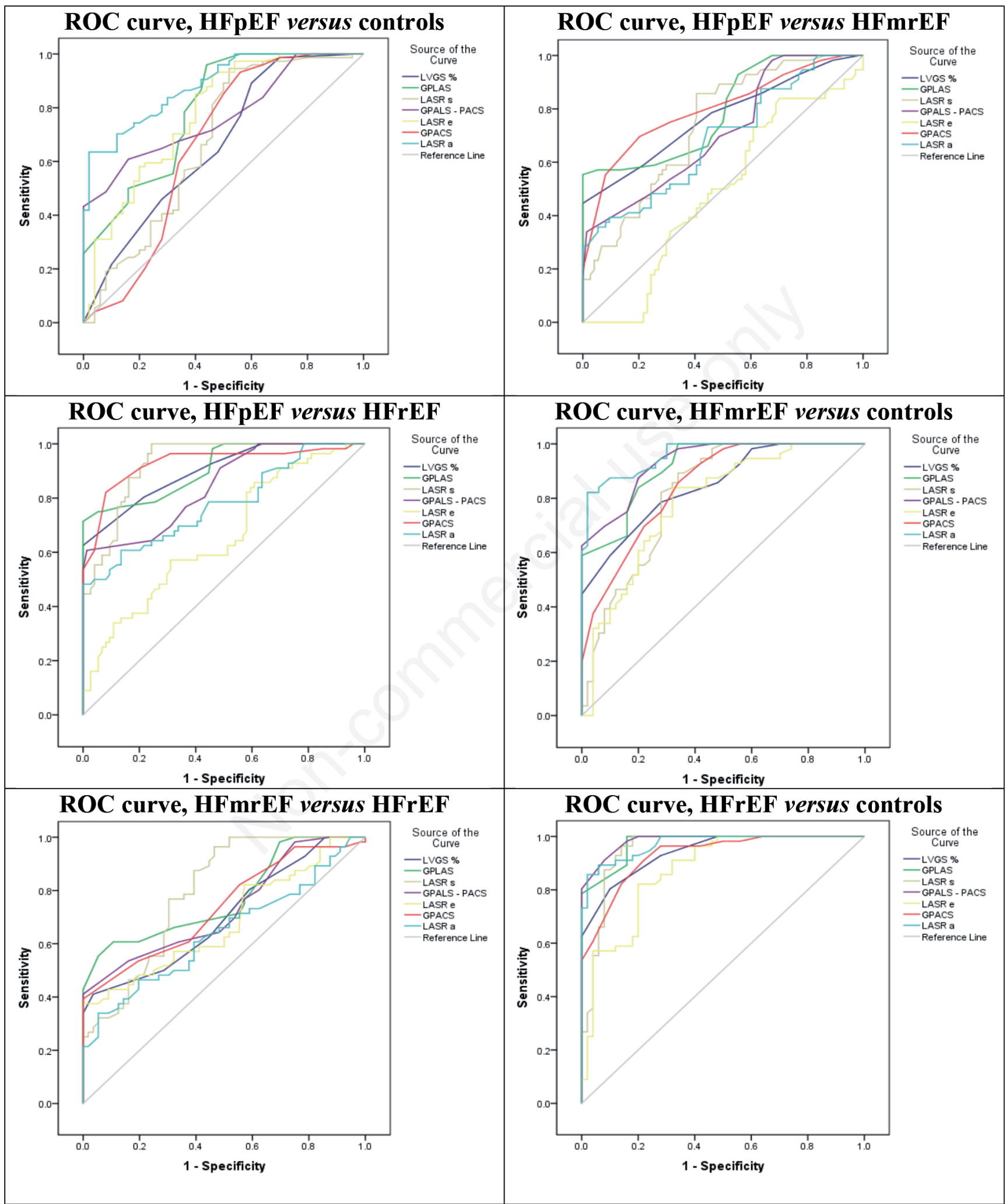

Figure 1. Receiver operating characteristic (ROC) curves for STE parameters of left atrial function in HFpEF, HFmrEF, HFrEF and controls. 
The strain imaging has enabled a deeper understanding of atrial function. Some authors have proposed GPALS as a better predictor of cardiovascular events than LAEF and LA function index [41]. The LA strain as a functional adaptive marker may provide valuable information on left atrium stiffness and indirectly estimate the LV end-diastolic pressure. It may identify atrial impairment at an early stage before dilatation occurs [42]. Kurt and colleagues [43] found significantly lower LA systolic strain levels in patients with HFpEF than in patients with LV diastolic dysfunction without HF. The reservoir strain is reduced in patients with SHF or DHF, furthermore SHF patients showed a more significant reduction in left atrium strain proportional to LVGS [44].

\section{Conclusions}

Early LA dysfunction in heart failure can be detected accurately and easily by speckle tracking technique that could be a promising independent tool to better understand of heart failure and its classification. The LVGS, GPALS, GPACS and GPALS-PACS are a novel independent parameter in early LA dysfunction to distinguish and predict HFpEF, HFmrEF and HFrEF.

\section{Study limitations}

Relatively small number of the study population. Time factor, there was no specific time interval between occurrences of HF and beginning of the study, non accurate selection of the patient group according to the cause of heart failure. The measurements of LA strain require good delineation of LA endocardial borders. This resulted in the exclusion of many patients from the analysis.

\section{References}

1. To AC, Flamm SD, Marwick TH, Klein AL. Clinical utility of multimodality LA imaging: assessment of size, function, and structure. JACC Cardiovasc Imaging 2011;4:788-98.

2. Cameli M, Lisi M, Mondillo S, et al. Left atrial longitudinal strain by speckle tracking echocardiography correlates well with left ventricular filling pressures in patients with heart failure. Cardiovasc Ultrasound 2010;8:14.

3. Cameli M, Lisi M, Giacomin E, et al. Chronic mitral regurgitation: Left atrial deformation analysis by two-dimensional speckle tracking echocardiography. Echocardiography 2011;28:327-34

4. Cameli M, Lisi M, Righini FM, Mondillo S. Novel echocardiographic techniques to assess left atrial size, anatomy and function. Cardiovasc Ultrasound 2012;10:4.

5. Ersboll M, Andersen MJ, Valeur N, et al. The prognostic value of left atrial peak reservoir strain in acute myocardial infarction is dependent on left ventricular longitudinal function and left atrial size. Circ Cardiovasc Imaging 2013;6:26-33.

6. Galli E, Fournet M, Chabanne C, et al. Prognostic value of left atrial reservoir function in patients with severe aortic stenosis: a 2D speckle-tracking echocardiographic study. Eur Heart J Cardiovasc Imaging 2016;17:533-41.

7. Freed BH, Daruwalla V, Cheng JY, et al. Prognostic utility and clinical significance of cardiac mechanics in heart failure with preserved ejection fraction: importance of left atrial strain. Circ Cardiovasc Imag 2016;9:e003754.

8. Kim DG, Lee KJ, Lee S, et al. Feasibility of twodimensional global longitudinal strain and strain rate imaging for the assessment of left atrial function: a study in subjects with a low probability of cardiovascular disease and normal exercise capacity. Echocardiography 2009;26:1179-87.

9. Todaro MC, Choudhuri I, Belohlavek M, et al. New echocardiographic techniques for evaluation of left atrial mechanics. Eur Heart J Cardiovasc Imaging 2012;13:973-84.

10. Ponikowski P, Voors AA, Anker SD, et al. 2016 ESC Guidelines for the diagnosis and treatment of acute and chronic heart failure: the Task Force for the diagnosis and treatment of acute and chronic heart failure of the European Society of Cardiology (ESC) developed with the special contribution of the Heart Failure Association (HFA) of the ESC. Eur Heart J 2016;37:2129-200.

11. Van Riet EE, Hoes AW, Limburg A, et al. Prevalence of unrecognized heart failure in older persons with shortness of breath on exertion. Eur J Heart Fail 2014;16:772-7.

12. Lam CS, Solomon SD. The middle child in heart failure: heart failure with midrange ejection fraction (40-50\%). Eur J Heart Fail 2014;16:1049-55.

13. Carluccio E, Biagioli P, Mengoni A, et al. Left atrial reservoir function and outcome in heart failure with reduced ejection fraction. The importance of atrial strain by speckle tracking echocardiography. Circ Cardiovasc Imaging 2018;11:e007696.

14. Nagueh SF, Smiseth OA, Appleton CP, et al. Recommendations for the evaluation of left ventricular diastolic function by echocardiography: an update from the American Society of Echocardiography and the European Association of Cardiovascular Imaging. Eur Heart J Cardiovasc Imaging 2016;17:1321-60.

15. Lang RM, Badano LP, Mor-Avi V, et al. Recommendations for cardiac chamber quantification by echocardiography in adults: an update from the American Society of Echocardiography and the European Association of Cardiovascular Imaging. Eur Heart J Cardiovasc Imaging 2015;16:233-71.

16. Ommen SR, Nishimura RA, Appleton CP, et al. Clinical utility of Doppler echocardiography and tissue Doppler imaging in the estimation of left ventricular filling pressures: A comparative simultaneous Doppler-catheterization study. Circulation 2000;102:1788-94.

17. Blume GG, Mcleod CJ, Barnes ME, et al. Left atrial function: physiology, assessment, and clinical implications. Eur J Echocardiogr 2011;12:421-30.

18. Ogawa K, Hozumi T, Sugioka K, et al. Automated assessment of left atrial function from time-left atrial volume curves using a novel speckle tracking imaging method. J Am Soc Echocardiogr 2009;22:63-9.

19. Di Salvo G, Al Bulbul Z, Issa Z, et al. Left ventricular mechanics after arterial switch operation: a speckle-tracking echocardiography study. J Cardiovasc Med (Hagerstown) 2016;17:217-24.

20. Saha SK, Anderson PL, Caracciolo G, et al. Global left atrial strain correlates with CHADS(2) risk score in patients with atrial fibrillation. J Am Soc Echocardiogr 2011;24:506-12.

21. Miyasaka Y, Tsujimoto S, Maeba H, et al. Left atrial volume by three-dimensional echocardiography: validation by $64-$ slice multidetector computed tomography. J Am Soc Echocardiogr 2011;24:680-6.

22. Mondillo S, Galderisi M, Mele D, et al. Echocardiography study group of the Italian Society of Cardiology (Rome, 
Italy). Speckle-tracking echocardiography: a new technique for assessing myocardial function. J Ultrasound Med 2011;30:71-83.

23. Wakami K, Ohte N, Asada K, et al. Correlation between left ventricular end-diastolic pressure and peak left atrial wall strain during left ventricular systole. J Am Soc Echocardiogr 2009;22:847-51.

24. Ponikowski P, Voors AA, Anker SD, et al. 2016 ESC Guidelines for the diagnosis and treatment of acute and chronic heart failure: The Task Force for the diagnosis and treatment of acute and chronic heart failure of the European Society of Cardiology (ESC) Developed with the special contribution of the Heart Failure Association (HFA) of the ESC. Eur Heart J 2016;37:2129-200.

25. Tokola H, Hautala N, Marttila M, et al. Mechanical loadinduced alterations in B-type natriuretic peptide gene expression. Can J Physiol Pharmacol 2001;79:646-53.

26. Iwanaga Y, Nishi I, Furuichi S, et al. B-type natriuretic peptide strongly reflects diastolic wall stress in patients with chronic heart failure: Comparison between systolic and diastolic heart failure. J Am Coll Cardiol 2006;47:742-8.

27. Modin D, Andersen DM, Biering-Sørensen T. Echo and heart failure: when do people needs an echo, and when do they need natriuretic peptides? Echo Res Pract 2018;5:R65-79.

28. Kemp CD, Conte JV. The pathophysiology of heart failure. Cardiovasc Pathol 2012;21:365-71.

29. Al Saikhan L, Hughes AD, Chung W, et al. Left atrial function in heart failure with mid-range ejection fraction differs from that of heart failure with preserved ejection fraction: a $2 \mathrm{D}$ speckle-tracking echocardiographic study. Eur Heart J Cardiovasc Imaging 2019;20:279-90.

30. Appleton CP, Galloway JM, Gonzalez MS, et al. Estimation of left ventricular filling pressures using two-dimensional and Doppler echocardiography in adult patients with cardiac disease. Additional value of analyzing left atrial size, left atrial ejection fraction and the difference in duration of pulmonary venous and mitral flow velocity at atrial contraction. J Am Coll Cardiol 1993;22:1972-82.

31. Vinereanu D, Nicolaides E, Tweddel AC, Fraser AG. 'Pure' diastolic dysfunction is associated with long-axis systolic dysfunction. Implications for the diagnosis and classification of heart failure. Eur J Heart Fail 2005;7:820-8.

32. Yip GWK, Zhang Q, Xie JM, et al. Resting global and regional left ventricular contractility in patients with heart failure and normal ejection fraction. Heart 2011;97:287-94.

33. Zakeri R, Moulay G, Chai Q, et al. Left atrial remodeling and atrioventricular coupling in a canine model of early heart failure with preserved ejection fraction. Circ Heart Fail 2016;9:e003238.

34. Melenovsky V, Hwang SJ, Redfield MM, et al. Left atrial remodeling and function in advanced heart failure with preserved or reduced ejection fraction. Circ Heart Fail 2015;8:295-303.

35. Singh A, Addetia K, Maffessanti F, et al. Strain categorization of LV diastolic dysfunction. JACC Cardiovasc Imaging 2017;10:735-43.

36. Aung SM, Guler A, Guler YF, et al. Left atrial strain in heart failure with preserved ejection fraction. Herz 2017;42:194-9.

37. Morris DA, Gailani M, Vaz Perez AF, et al. Left atrial systolic and diastolic dysfunction in heart failure with normal left ventricular ejection fraction. $\mathrm{J}$ Am Soc Echocardiogr 2011;24:651-62.

38. King JB, Azadani PN, Suksaranjit PF, et al. Left atrial fibrosis and risk of cerebrovascular and cardiovascular events in patients with atrial fibrillation. J Am Coll Cardiol 2017;70:1311-21.

39. Bech-Hanssen O, Pergola V, Al-Admawi M, et al. Atrial function in heart transplant recipients operated with the bicaval technique. Scand Cardiovasc J 2016;50:42-51.

40. Cameli M, Sparla S, Losito M, et al. Correlation of left atrial strain and doppler measurements with invasive measurement of left ventricular end-diastolic pressure in patients stratified for different values of ejection fraction. Echocardiography 2016;33:398- 405

41. Sargento L, Vicente Simões A, Longo S, et al. Left atrial function index predicts long-term survival in stable outpatients with systolic heart failure. Eur Heart J Cardiovasc Imaging 2017;18:119-27.

42. Bowman AW, Kovács SJ. Assessment and consequences of the constant volume attribute of the four-chambered heart. Am J Physiol Heart Circulat Physiol 2003;285:H2027-33.

43. Kurt M, Wang J, Torre-Amione G, Nagueh SF. Left atrial function in diastolic heart failure. Circ Cardiovasc Imaging 2009;2:10-5.

44. Frydas A, Morris DA, Belyavskiy E, et al. Left atrial strain as sensitive marker of left ventricular diastolic dysfunction in heart failure. ESC Heart Fail 2020;7:1956-65. 